\title{
Induction of apoptosis by diphenyldifluoroketone in osteogenic sarcoma cells is associated with activation of caspases
}

\author{
SEOK-JIN YANG ${ }^{1 *}$, SEUL AH LEE $^{1 *}$, MIN-GYEONG PARK ${ }^{1}$, JAE-SUNG KIM $^{1,2}$, SUN-KYOUNG YU ${ }^{1}$, \\ CHUN SUNG KIM ${ }^{1}$, JIN-SOO KIM ${ }^{1}$, SU-GWAN KIM ${ }^{1,2}$, JI-SU OH ${ }^{1,2}$, HEUNG-JOONG KIM ${ }^{1}$, \\ HONG SUNG CHUN ${ }^{3}$, YONG HWAN KIM ${ }^{4}$ and DO KYUNG KIM ${ }^{1,2}$ \\ ${ }^{1}$ Oral Biology Research Institute, ${ }^{2}$ Regional Innovation Center for Dental Science and Engineering, \\ ${ }^{3}$ Department of Biotechnology, Chosun University, Gwangju 501-759; ${ }^{4}$ Korea Institute of Planning and Evaluation \\ for Technology in Food, Agriculture Forestry and Fisheries, Gyeonggi-do 431-810, Republic of Korea
}

Received December 28, 2013; Accepted February 18, 2014

DOI: 10.3892/or.2014.3066

\begin{abstract}
The aim of the present study was to investigate and compare the effects of diferuloylmethane (curcumin) and diphenyldifluoroketone (EF-24) on cell growth and apoptosis induction in human osteogenic sarcoma cells. This was examined by MTT assay, nuclear DAPI staining, caspaseactivation assay, flow cytometry analysis and immunoblotting in Saos2 human osteogenic sarcoma cells. Curcumin and EF-24 inhibited the growth of Saos2 cells in a dose-dependent manner. The apparent potency of EF-24 was more than 3-fold higher that of curcumin. Treatment with curcumin or EF-24 resulted in nuclear condensation and fragmentation in the cells. The caspase-3/-7 activities were detected in living cells treated with curcumin or EF-24. Flow cytometry showed that the rate of apoptosis was increased by curcumin and EF-24 compared to the control. Curcumin and EF-24 promoted the proteolytic cleavages of procaspase-3/-7/-8/-9 with increases in the amount of cleaved caspase-3/-7/-8/-9. The curcumin- or EF-24-induced apoptosis in the Saos2 cells was mediated by the expression of Fas and activation of caspase-8, caspase-3 and poly(ADP-ribose) polymerase. Immunoblotting revealed the Bid and Bcl-2 proteins to be downregulated, and truncatedBid, Bax and p53 proteins to be upregulated by curcumin and EF-24. Curcumin and EF-24 increased the Bax/Bcl-2 ratio significantly. These results suggest that the curcumin and EF-24 inhibit cell proliferation and induce apoptotic cell death in Saos2 human osteogenic sarcoma cells via both the mitochondria-mediated intrinsic pathway and the death
\end{abstract}

Correspondence to: Professor Do Kyung Kim, Department of Oral Physiology, Chosun University School of Dentistry, 375 Seosuk-dong, Dong-gu, Gwangju 501-759, Republic of Korea E-mail:kdk@chosun.ac.kr

${ }^{*}$ Contributed equally

Key words: diferuloylmethane, diphenyldifluoroketone, cell death, apoptosis, osteosarcoma, anticancer activity receptor-mediated extrinsic pathway, and may have potential properties for anti-osteosarcoma drug discovery.

\section{Introduction}

Osteosarcoma is the most common primary bone malignant tumor, affecting primarily children and young adolescents (1). Despite the advances in multimodality treatments, the combination of neoadjuvant chemotherapy plus surgery have increased the survival rates markedly from 20 to $65 \%$ since the 1980 s, but the progress has been considerably slow over the past 20 years $(2,3)$. Therefore, an understanding of the molecular mechanisms of osteosarcoma is one of the most important issues for treatment. New therapeutic strategies are necessary to increase the survival rates in patients with osteosarcoma (4).

In recent years, there has been a global trend toward the importance of naturally occurring phytochemicals in plants for the prevention and treatment of human diseases. Several of these phytochemicals have shown potential as cancer chemopreventive or therapeutic agents in the human body (5). Most of these bioactive phytochemicals exert their cancer chemotherapeutic activity by blocking cell cycle progression and triggering apoptotic cell death (5). Therefore, the induction of apoptosis in cancer cells is an important indicator of the cancer treatment response when employing a bioactive substance to reduce and control human mortality due to cancer $(6,7)$.

Apoptosis, which is a major form of programmed cell death, plays an important role in regulating tissue development and homeostasis in eukaryotes (8). Apoptosis can occur via a death receptor-dependent extrinsic or a mitochondria-dependent intrinsic pathway, and apoptosis is induced by a treatment with chemotherapeutic agents $(9,10)$.

Diferuloylmethane (curcumin), which is a constituent of turmeric powder derived from the rhizome of Curcuma longa, has anti-inflammatory, antimicrobial, anti-oxidative, immunomodulating and anti-atherogenic properties $(11,12)$. Furthermore, many studies have reported that curcumin has chemopreventive and antiproliferative activity in many human cancers $(12,13)$. Of particular interest is that curcumin is also pharmacologically safe as it is a naturally occurring 
compound (14). However, natural curcumin has limited use due to its poor intestinal absorption and low bioavailability (15). On the other hand, it is an excellent compound for the design of more effective analogs. One monoketone analog in particular, diphenyldifluoroketone (EF-24), is effective in anticancer screens (16). EF-24 was reported to inhibit the growth of human breast tumor xenografts in a mouse model with relatively low toxicity and at a much lower dose than that of curcumin (16). Although it was shown to reduce the cancer cell viability, its action mechanisms remain to be determined. In addition, the effects of EF-24 and curcumin on osteosarcoma are unclear.

The present study examined the effects of EF-24 and curcumin on cell growth and the mechanism of cell death elicited by EF-24 and curcumin in Saos2 human osteogenic sarcoma cells. The study reports for the first time that the induction of apoptotic cell death by EF-24 is associated with the activation of caspases in osteogenic sarcoma cells.

\section{Materials and methods}

Materials. Saos2 human osteogenic sarcoma cells were provided by the American Type Culture Collection (ATCC, Rockville, MD, USA). EF-24 and curcumin were supplied by Sigma (St. Louis, MO, USA). Anti-cleaved caspase-3/-7/-8/-9, poly(ADP-ribose) polymerase (PARP), Fas, Bid, Bax, Bcl-2, p53 and $\beta$-actin antibodies were purchased from Cell Signaling Technology, Inc. (Danvers, MA, USA). The cell-permeable fluorogenic substrate PhiPhiLux- $\mathrm{G}_{1} \mathrm{D}_{2}$ was purchased from OncoImmunin, Inc. (Gaithersburg, MD, USA). All other analytical reagents purchased were of analytical grade.

Cell cultures. The Saos 2 human osteogenic sarcoma cells were grown in DMEM supplemented with 10\% fetal bovine serum (FBS) (17). The cells were maintained as monolayers in plastic culture plates at $37^{\circ} \mathrm{C}$ in a humidified atmosphere containing $5 \% \mathrm{CO}_{2}$.

MTT assay. The Saos 2 cells were seeded at a concentration of $5 \times 10^{3}$ cells/well in 24-well plates. After 24-h growth, the cells were treated with EF-24 or curcumin at various concentrations for $24 \mathrm{~h}$. The cell viability was assessed using an MTT assay.

Nuclear staining with DAPI. Nuclear staining with DAPI (40,60-diamidino-2-phenylindole) was performed to examine the level of apoptosis. The Saos 2 cells were cultured in 24-well plates at a seeding density of $5 \times 10^{3}$ cells/well. After $24-\mathrm{h}$ growth, the cells were treated with $10 \mu \mathrm{M} \mathrm{EF-24}$ or $30 \mu \mathrm{M}$ curcumin for $24 \mathrm{~h}$. The treated Saos 2 cells were fixed with $1 \%$ paraformaldehyde for $30 \mathrm{~min}$ at room temperature and washed twice with PBS. The cells were permeated with icecold ethanol for $5 \mathrm{~min}$ at room temperature and washed twice with PBS. The fixed cells were stained with DAPI $(300 \mathrm{nM})$ for $5 \mathrm{~min}$ at room temperature in the dark, washed twice with PBS and examined by fluorescent inverted microscopy (IX71; Olympus, Tokyo, Japan).

Determination of caspase activation. The activity of caspase-3/-7 was determined using the cell-permeable fluorogenic substrate, PhiPhiLux- $\mathrm{G}_{1} \mathrm{D}_{2}$, according to the

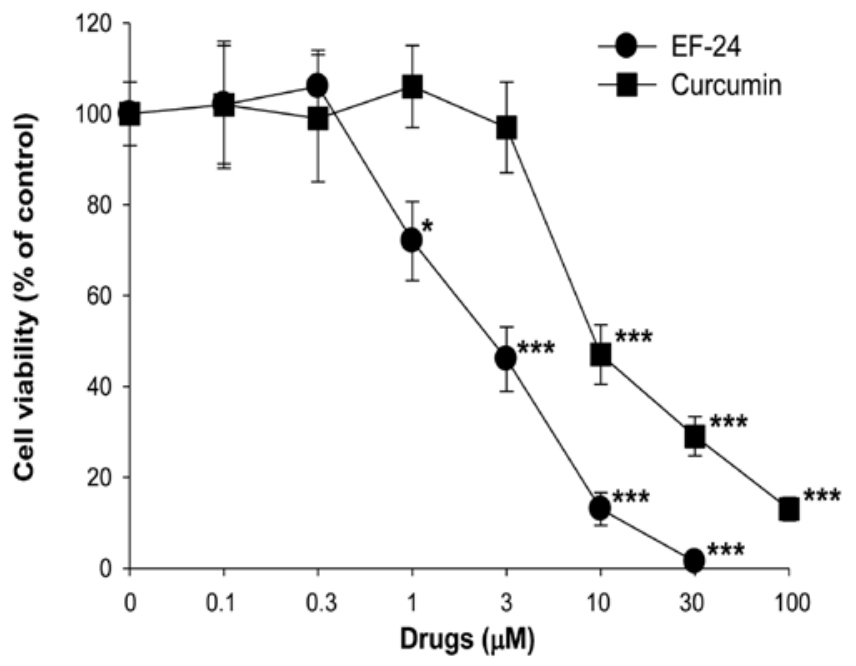

Figure 1. Concentration-dependent effects of EF-24 and curcumin on the cell viability in Saos 2 human osteogenic sarcoma cells. The Saos 2 cells were treated with various concentrations of EF-24 (circle) and curcumin (square) or without EF-24 and curcumin for $24 \mathrm{~h}$. The cell viabilities were determined by MTT assays. The percentage of cell viability was calculated as a ratio of A570 nm of the EF-24- or curcumin-treated cells and untreated control cells. Each data point represents the mean \pm SEM of six experiments. ${ }^{*} \mathrm{P}<0.05$ vs. control and ${ }^{* * * *} \mathrm{P}<0.001$ vs. control (the control cells measured in the absence of EF-24 or curcumin).

manufacturer's instructions. The cells were treated with 0 and $10 \mu \mathrm{M} \mathrm{EF}-24$ or 0 and $30 \mu \mathrm{M}$ curcumin for $24 \mathrm{~h}$ and incubated with PhiPhiLux- $\mathrm{G}_{1} \mathrm{D}_{2}$. The activity of caspase-3/-7 was examined by fluorescence microscopy (IX71; Olympus).

Annexin V-fluorescein isothiocyanate (V-FITC), propidium iodide (PI) and flow cytometric analysis. The Saos 2 cells were cultured in $100-\mathrm{mm}$ tissue-culture dishes at a density of $1 \times 10^{6}$ cells/dish for $24 \mathrm{~h}$ and were treated with $10 \mu \mathrm{M} \mathrm{EF}-24$ or $30 \mu \mathrm{M}$ curcumin for $24 \mathrm{~h}$. The cells were washed twice in phosphatebuffered saline and resuspended in the binding buffer (BD Biosciences, San Diego, CA, USA). Annexin V-FITC and 7-amino-actinomycin D (BD Biosciences) were added to the cells, which were then incubated in the dark for $15 \mathrm{~min}$, and resuspended in $400 \mu \mathrm{l}$ of binding buffer. The cells were analyzed using a fluorescence activated cell sorting Calibur flow cytometer (Becton-Dickinson, San Jose, CA, USA). Data analysis was performed using standard CellQuest software (Becton-Dickinson).

Immunoblotting. The Saos2 cells were treated with EF-24 or curcumin for $24 \mathrm{~h}$. Immunoblotting was performed using a minor modification of the method described elsewhere (18). Anti-cleaved caspase-3/-7/-8/-9, PARP, Fas, Bid, tBid, Bax, Bcl-2 and p53 antibodies (Cell Signaling Technology) were used as the primary antibodies.

Data analysis. All experiments were performed at least in triplicate. The results are presented as the mean \pm SEM. The statistical significance was analyzed using a Student's t-test for the two group comparison and one-way analysis of the variance for the multi-group comparisons. A P-value $<0.05$ was considered to indicate a statistically significant difference. 

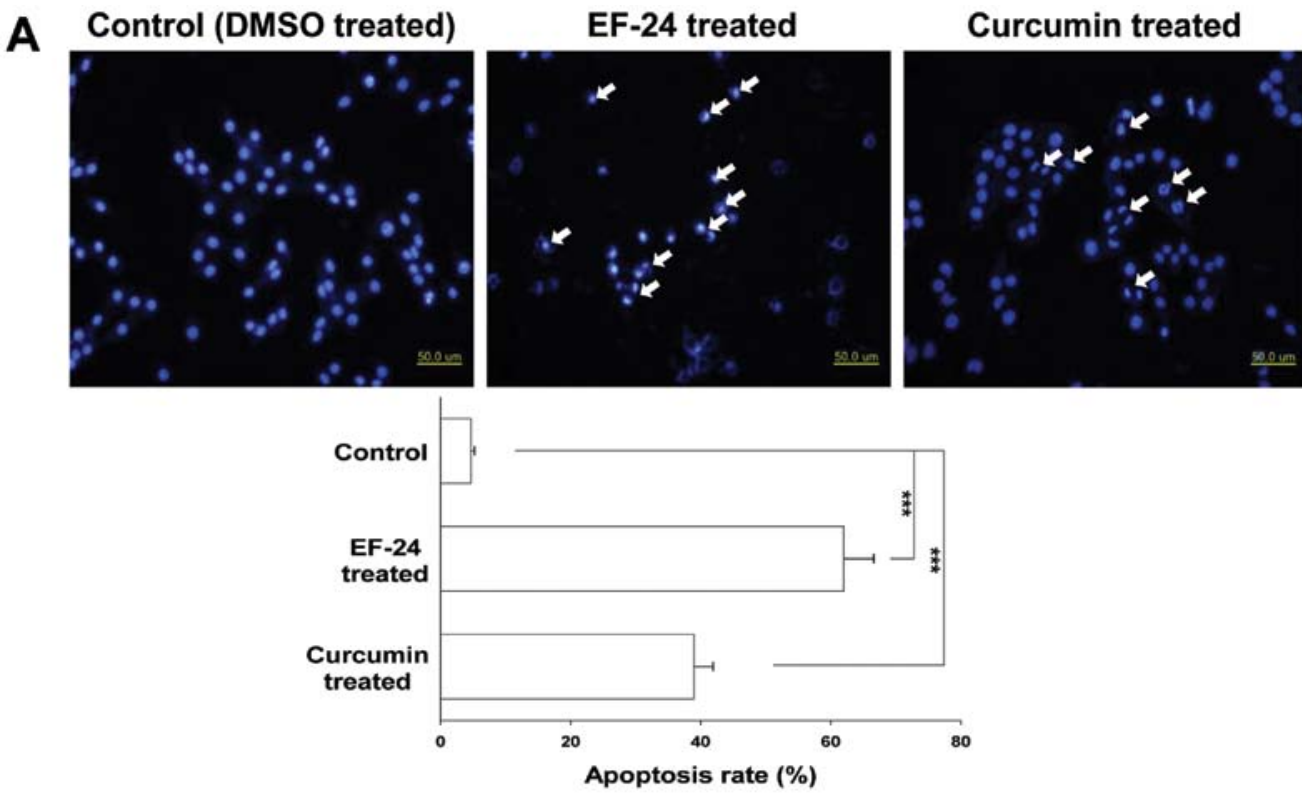

B

Caspase-3/-7 activity by EF-24 and Curcumin treatment
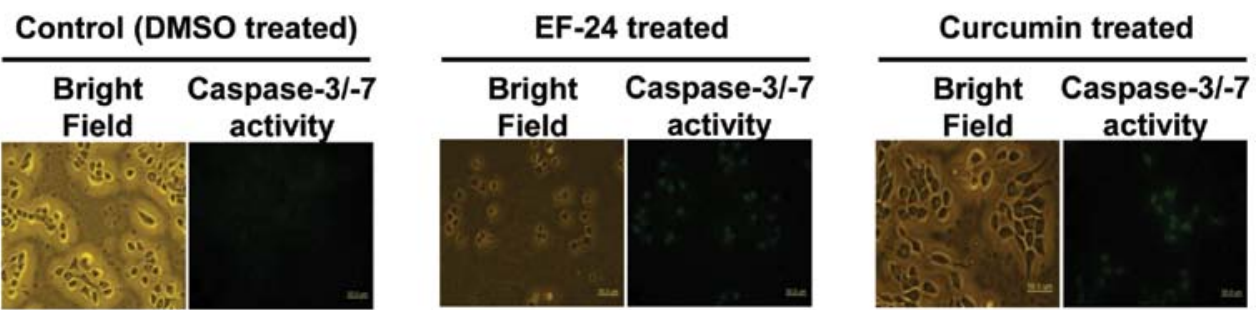

Figure 2. Induction of apoptosis by EF-24 and curcumin in Saos2 cells. (A) Changes in nuclear morphology by EF-24 and curcumin. The cells were treated with $10 \mu \mathrm{M} \mathrm{EF-24}$ or $30 \mu \mathrm{M}$ curcumin for $24 \mathrm{~h}$. Representative fluorescence photomicrographs show the nuclei morphology of Saos 2 cells. The arrows indicate chromatin condensation, reduced nuclear size and nuclear fragmentation typically observed in apoptotic cells. The lower panel shows that the percentage of apoptotic cells was calculated as the ratio of apoptotic cells to total cells. ${ }^{* * *} \mathrm{P}<0.001$ vs. control (the control cells measured in the absence of EF-24 or curcumin). (B) Activation of caspase-3/-7 by EF-24 or curcumin treatment in living Saos 2 cells. The cells were treated with $10 \mu \mathrm{M}$ EF- 24 or $30 \mu \mathrm{M}$ curcumin for $24 \mathrm{~h}$ and the specific cell-permeable substrate PhiPhiLux $\mathrm{G}_{1} \mathrm{D}_{2}$ was then added. Active caspase-3/-7 was visualized by fluorescence microscopy.

\section{Results}

Cytotoxic effects of EF-24 and curcumin on Saos 2 cells. The Saos2 cells were treated with EF-24 and curcumin at various concentrations for $24 \mathrm{~h}$, and were analyzed by MTT assay. As shown in Fig. 1, treatment of curcumin at doses from 0.1 to $3 \mu \mathrm{M}$ did not significantly affect the cell viability of the Saos 2 cells, but curcumin at 10,30 and $100 \mu \mathrm{M}$ reduced Saos2 cell viability. When the cells were treated with EF-24 for $24 \mathrm{~h}, \mathrm{EF}-24$ inhibited the growth of cells in a dose-dependent manner, suggesting that EF-24 induces Saos2 cell death (Fig. 1). The $\mathrm{IC}_{50}$ values of EF-24 and curcumin on Saos2 cell viability after a 24 -h treatment were $2.7 \pm 0.3$ and $9.7 \pm 1.4 \mu \mathrm{M}$, respectively. The apparent potency of EF-24 was $>3$-fold higher that of curcumin. More importantly, the effects were observed at an EF-24 concentration $<3 \mu \mathrm{M}$, a dose at which curcumin had no significant effects on cell proliferation, indicating the higher potency of EF-24.

Changes in the nuclear morphology by EF-24 and curcumin . The nuclear morphological changes were assessed by DAPI staining. As shown in Fig. 2A, the nuclei of the control Saos2 cells (control) had a normal regular and oval shape. Treatment with $10 \mu \mathrm{M}$ EF-24 or $30 \mu \mathrm{M}$ curcumin for $24 \mathrm{~h}$ resulted in nuclear condensation and fragmentation, which are the characteristics of apoptosis (Fig. 2A, upper panel). As quantified in Fig. 2A (lower panel), EF-24 and curcumin increased the apoptotic rate of Saos2 cells significantly to $62.0 \pm 4.6$ and $39.1 \pm 2.9 \%$, respectively.

Activation of caspase-3/-7 by EF-24 and curcumin. The activation of caspase-3/-7 in the EF-24- or curcumin-treated Saos 2 cells was confirmed by fluorescence microscopy using a fluorogenic substrate. As shown in Fig. 2B, both the EF-24 and curcumin treatment led to the activation of caspase-3/-7 in the living Saos 2 cells.

Apoptosis induction of Saos 2 cells by EF-24 and curcumin. To determine if EF-24- or curcumin-induced cell death is associated with the induction of apoptosis, the Saos 2 cells were stimulated with $10 \mu \mathrm{M} \mathrm{EF}-24$ or $30 \mu \mathrm{M}$ curcumin for $24 \mathrm{~h}$ and then co-stained with Annexin V-FITC, an apoptotic marker, and PI, a necrotic marker. The percentage of Annexin V-FITCpositive cells at the stage of apoptosis was increased to 64.1 and $43.9 \%$ by EF-24 and curcumin, respectively, compared to the control (Fig. 3). 

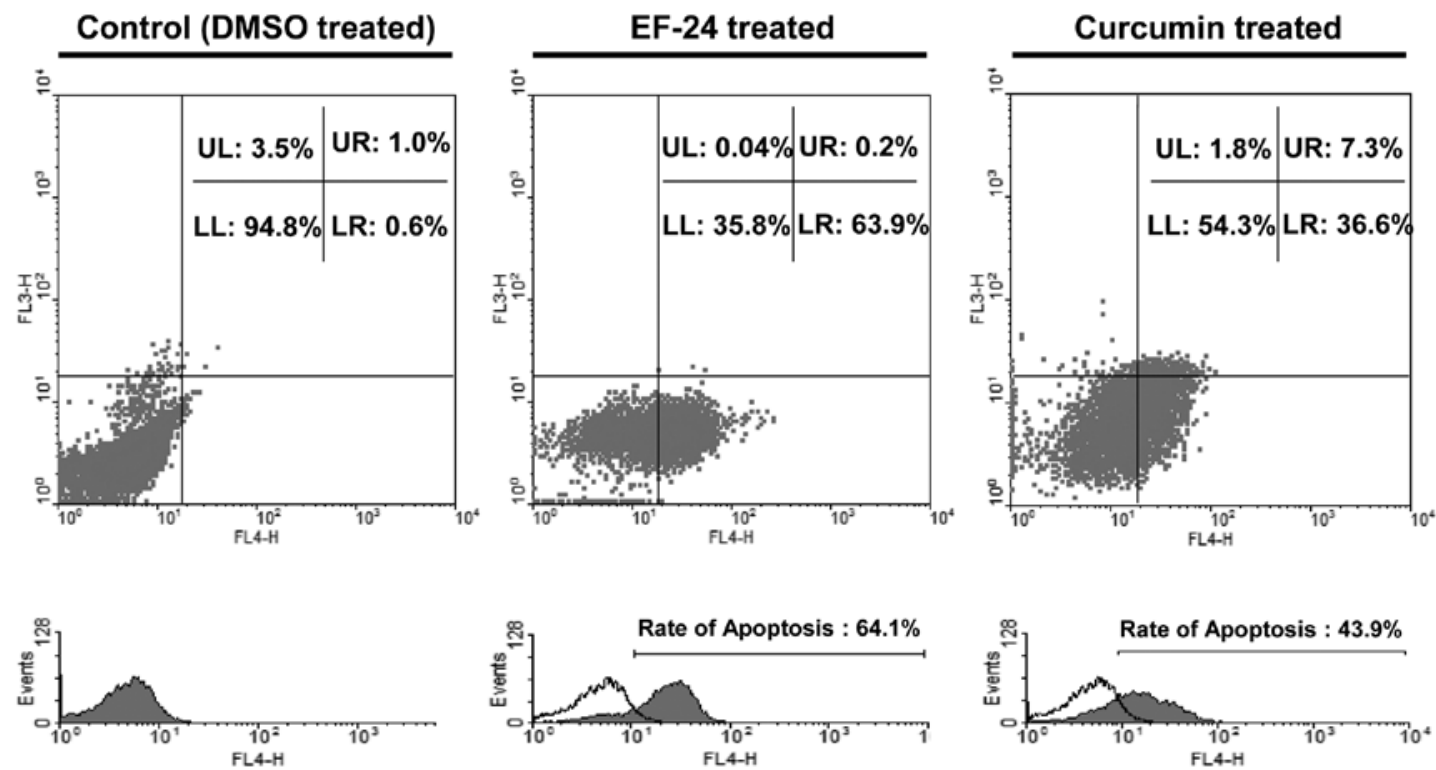

Figure 3. Apoptotic population induction of Saos2 cells by EF-24 and curcumin. To identify the EF-24- or curcumin-induced Saos 2 cell apoptosis, FACS analysis was performed by Annexin V and PI staining. The Saos 2 cells were cultured in complete medium, and stimulated with $10 \mu \mathrm{M}$ EF-24 or $30 \mu \mathrm{M}$ curcumin for $24 \mathrm{~h}$. After stimulation, the cells were analyzed by flow cytometry. LL, lower left (normal); UL, upper left (necrosis); LR, lower right (early phase of apoptosis); UR, upper right (late phase of apoptosis).

A

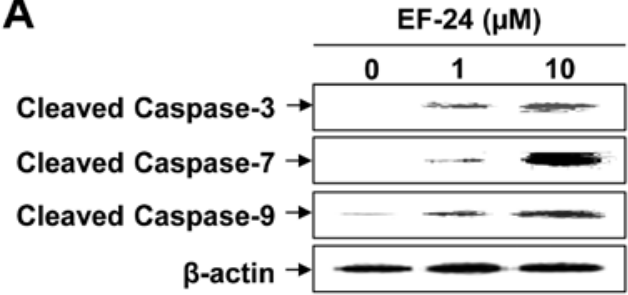

B

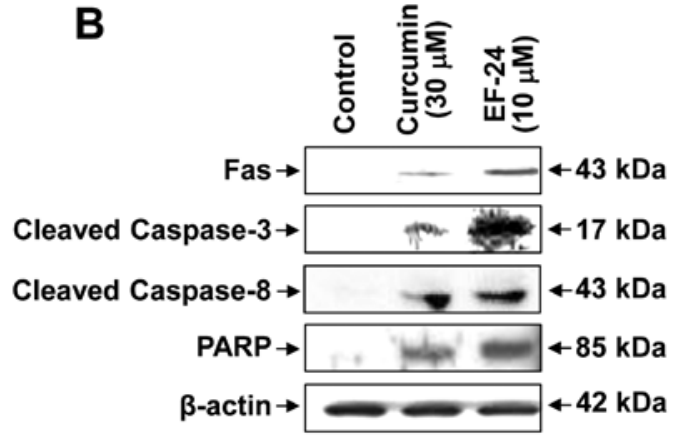

\section{Curcumin $(\mu \mathrm{M})$}

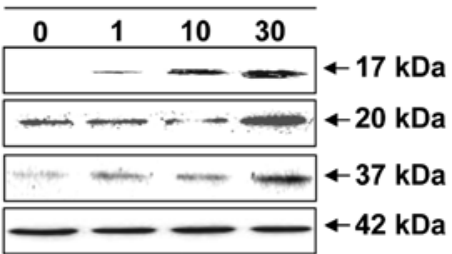

C

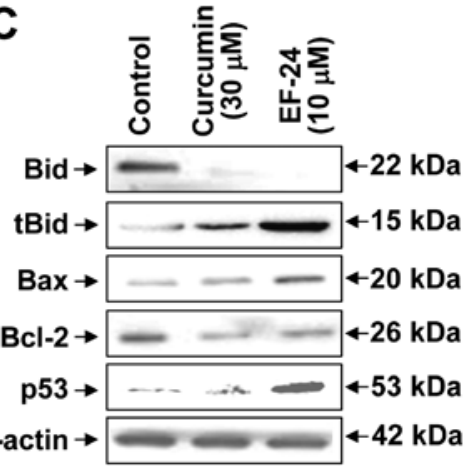

Figure 4. EF-24 or curcumin-induced apoptosis mediated by expression and activation of caspases in Saos 2 cells. (A) Proteolytic cleavage of caspase-3/-7/-9 by EF-24 or curcumin treatment. The activity of cleaved caspase-3/-7/-9 by EF-24 or curcumin was measured in Saos2 cells. The cells were treated with 0,1 and $10 \mu \mathrm{M} \mathrm{EF}-24$ or $0,1,10$ and $30 \mu \mathrm{M}$ curcumin for $24 \mathrm{~h}$. The cell lysate was prepared and analyzed by immunoblotting as described in Materials and methods. (B) Activation of the extrinsic apoptosis signaling pathway by EF-24 and curcumin. The activity of extrinsic apoptosis signaling pathway in Saos 2 cells by EF-24 or curcumin was measured. The Saos2 cells were stimulated with 0 and $10 \mu \mathrm{M} \mathrm{EF-24}$ or 0 and $30 \mu \mathrm{M}$ curcumin for $24 \mathrm{~h}$, harvested, and lysed using a cell lysis buffer. (C) Activation of p53 and regulation of Bid, tBid, Bax and Bcl-2 levels by EF-24 and curcumin. The activities of p53, Bid, tBid, Bax and Bcl-2 by EF-24 or curcumin were measured in Saos 2 cells.

Activation of caspases by EF-24 and curcumin. The levels of cleaved caspase-3/-7/-9 were examined by immunoblotting as caspase-3/-7/-9 are effector caspases of apoptotic cell death (19). Treatment with 1 and $10 \mu \mathrm{M}$ EF-24 or 1, 10 and $30 \mu \mathrm{M}$ curcumin for $24 \mathrm{~h}$ promoted the proteolytic cleavage of procaspase-3/-7/-9 in Saos 2 cells, with increases in the amount of cleaved caspase-3/-7/-9 (Fig. 4A).
Apoptosis mediated via Fas/PARP axis by EF-24 and curcumin. To determine how EF-24 or curcumin induce the apoptosis of Saos2 cells, immunoblotting was performed to measure the expression of the apoptotic genes at the protein level. As shown in Fig. 4B, Fas, which is an apoptotic ligand that triggers the extrinsic apoptotic pathway in Saos2 cells $(20,21)$, was induced significantly by either EF-24 or 
curcumin. Subsequently, the cleaved caspase- 3 and -8 , which are the downstream targets of Fas, were induced by EF-24 or curcumin. Cleaved PARP was increased by EF-24 or curcumin compared to the control.

Apoptosis-related signal pathways by EF-24 and curcumin The level of several proteins that are highly relevant to understanding the apoptotic signaling pathways in Saos 2 cells by EF-24 or curcumin was measured by immunoblot analysis. The level of Bid and Bcl-2 protein expressions in Saos2 cells stimulated with $10 \mu \mathrm{M}$ EF-24 or $30 \mu \mathrm{M}$ curcumin for $24 \mathrm{~h}$ decreased (Fig. 4C). On the other hand, the treatment of Saos2 cells with EF-24 or curcumin increased the level of truncatedBid (tBid), Bax and p53 protein expression (Fig. 4C).

\section{Discussion}

Recent studies have shown that the chemicals derived from natural materials can elicit chemopreventive and therapeutic effects $(5,22)$. This effect was reported to alter many factors associated with the cell cycle and to induce apoptotic cell death $(5,23)$. Finding new anticancer agents that can kill cancerous cells with minimal toxicity is critical. Curcumin has been extracted from the dried ground rhizome of the perennial herb, Curcuma longa. Several studies have suggested that curcumin induces cell cycle arrest and apoptosis in a variety of cancer cells $(12,13)$. On the other hand, the design of more effective analogs is needed due to the poor intestinal absorption and low bioavailability of curcumin (15). EF-24, a monoketone analog of curcumin, is efficacious in anticancer screens and was reported to inhibit the growth of human breast tumor xenografts in a mouse model (16). Although EF-24 can reduce cancer cell viability, the mechanisms of action are unclear, and the effects of EF-24 and curcumin on osteosarcoma have not been established. Osteosarcoma is the most common type of malignant bone tumor, in which the neoplastic mesenchymal cells show evidence of osteoid production (18). The present study examined the cytotoxic effects of EF-24, and the mechanism of cell death exhibited by EF-24 in Saos2 human osteogenic sarcoma cells was assessed. The results suggest that EF-24, a novel curcumin analog, possesses considerable promise as an anti-osteosarcoma therapeutic.

An MTT assay showed that the treatment of curcumin from 0.1 to $3 \mu \mathrm{M}$ did not significantly affect the cell viability of Saos 2 cells, but curcumin at 10,30 and $100 \mu \mathrm{M}$ reduced Saos2 cell viability (Fig. 1). EF-24 inhibited the growth of Saos 2 cells in a concentration-dependent manner (Fig. 1). This corresponded to the results of EF-24 and curcumin, which have anticancer effects via the suppression of cancer cell growth in many types of cancer cells $(12,13)$. In addition, the apparent potency of EF-24 was $>3$-fold higher that of curcumin. This suggests that EF-24 and curcumin are cytotoxic to osteosarcoma cells with EF-24 having higher potency. In addition, these results suggest that it has potential value for anticancer drug discovery.

Apoptosis is an important way of maintaining cellular homeostasis between cell division and cell death (8). The induction of apoptosis in cancer cells is a useful strategy for anticancer drug development (24). Therefore, many studies have screened plant-derived compounds for their effects on apoptosis (5). In the present study, treatment with EF-24 and curcumin induced nuclear condensation and fragmentation and the activation of the caspase-3/-7 in living Saos 2 cells, suggesting apoptotic cell death (Fig. 2). These results indicate that EF-24 and curcumin inhibit the growth of Saos 2 cells by activating cell apoptosis.

Curcumin and EF-24 were evaluated for their apoptosisinducing activities by the flow cytometry of Saos 2 cells stained with Annexin V-FITC and PI. Exposure of the membrane phospholipid, phosphatidylserine, to the external cellular environment is one of the earliest markers of apoptotic cell death $(25,26)$. Annexin V is a calcium-dependent phospholipid binding protein with high affinity to phosphatidylserine expressed on the cell surface (25). PI does not enter a cell with an intact cell-membrane and has been used to differentiate among early apoptotic (Annexin V-positive, PI-negative), late apoptotic (Annexin V-, PI-double positive) and necrotic cell death (Annexin V-negative, PI-positive) (25). In the present study, the ratio of early apoptotic Saos 2 cells (lower right) was increased after a treatment with $10 \mu \mathrm{M} \mathrm{EF}-24$ (from 0.6 to $63.9 \%$ ) or $30 \mu \mathrm{M}$ curcumin (from 0.6 to $36.6 \%$ ) (Fig. 3). These results showed that most of the cytotoxic activity of curcumin and EF-24 against Saos2 cells is due to the induction of apoptotic cell death.

The activation of a family of intracellular cysteine proteases, known as caspases, plays an important role in the initiation and execution of apoptosis induced by a range of stimuli (27). Among the caspases identified in mammalian cells, caspase-3/-7/-8/-9 can serve as the effector caspases of apoptotic cell death (27). Caspase-3/-7/-8/-9 are synthesized as inactive proenzymes, which require proteolytic activation to cleaved enzymes (of sizes 17, 20, 43 and $37 \mathrm{kDa}$, respectively) (27). The results of the present study showed that the amount of cleaved caspase-3/-7/-8/-9 in Saos2 cells was increased after the EF-24 or curcumin treatment (Fig. 4A and B). These results suggest that EF-24 and curcumin induce apoptotic cell death through both the mitochondriamediated intrinsic pathway and death receptor-mediated extrinsic pathway by the activation of caspases-3/-7/-8/-9 in Saos 2 cells.

The factor associated suicide ligand (Fas), which is an important regulatory factor of apoptosis, initiates the death receptor-mediated extrinsic apoptotic pathway through the activation of caspase- $8,-3$ and PARP, sequentially, after binding with the receptor FasR spanned on the surface of the target cells $(20,21)$. In the present study, the expression of Fas was upregulated significantly by EF-24 or curcumin in Saos2 cells (Fig. 4B). Subsequently, Fas upregulated by EF-24 or curcumin triggers a caspase cascade, which then results in the activation of apoptotic factors, including cleaved caspase- 8 and -3. Finally, activated caspase-3 cleaved its major substrate, PARP, leading to consequent apoptosis. Therefore, these results suggest that EF-24- or curcumin-induced apoptosis in Saos 2 cells is mediated by the death receptor-mediated extrinsic apoptotic pathway via the Fas/PARP axis.

Next, we assessed the effects of EF-24 and curcumin on the expression of p53, Bid, tBid, Bax and Bcl-2 in Saos 2 cells. The molecular biological pathways underlying the inhibition of cancerous growth typically involve tumor suppressors, such as p53 (8,28-30). The level of p53 is consistent in normal 
cells and the protein becomes phosphorylated during cellular stress, which then interacts with mouse double minute 2 (MDM2), resulting in apoptosis $(8,28-31)$. In the present study, the level of p53 was higher in the Saos 2 cells stimulated with EF-24 or curcumin than in the control (Fig. 4C), indicating that p53 may mediate the EF-24- or curcumin-induced apoptosis of Saos 2 cells. The pro-apoptotic proteins, Bid, tBid and Bax, and the anti-apoptotic mitochondrial protein, Bcl-2, are important regulators of cytochrome $c$ release from the mitochondria $(32,33)$. The Bcl-2 family is localized to the mitochondrial membrane and modulates apoptosis by permeabilizing the mitochondrial membrane, leading to the release of cytochrome $c$ (34). In the present study, treatment of Saos2 cells with EF-24 or curcumin decreased the level of Bid and increased the level of tBid (Fig. 4C). Caspase- 8 was reported to truncate $\mathrm{Bid}$, and $\mathrm{tBid}$ could activate the mitochondrial pathway (35). In addition, treatment of Saos2 cells with EF-24 or curcumin decreased the level of Bcl-2 but increased the level of Bax (Fig. 4C). The Bax/Bcl-2 ratio is one of the indices of the intrinsic mechanism of apoptosis in the mitochondria (36). EF-24 or curcumin-induced apoptosis appears to involve Bax/Bcl-2 signal transduction since EF-24 and curcumin increased this ratio in Saos 2 cells. Therefore, EF-24 and curcumin are suggested to induce apoptosis in Saos 2 cells involving both the mitochondrial- and death receptor-signal transduction pathways. On the other hand, the mechanisms of apoptosis induced by EF-24 and curcumin in Saos 2 cells are not fully understood. Further studies are required to examine the precise cellular and molecular mechanisms of apoptosis induced by EF-24 and curcumin.

In conclusion, these in vitro results suggest that EF-24 and curcumin inhibit cell proliferation and induce apoptotic cell death in Saos2 human osteogenic sarcoma cells through both the mitochondria-mediated intrinsic pathway and the death receptor-mediated extrinsic pathway. Moreover, EF-24 was $>3$-fold more potent than curcumin. Overall, EF-24 can be a model compound for the further development of natural product-derived anti-osteosarcoma agents. On the other hand, to elaborate on this nascent possibility, a further study of its activity including in vivo and the purification of bioactive compounds is currently being conducted.

\section{Acknowledgements}

The present study was supported by a research fund from Chosun University, 2013.

\section{References}

1. Daiy H, Huangy Y, Li Y, Meng G, Wang Y and Guo QN: TSSC3 overexpression associates with growth inhibition, apoptosis induction and enhances chemotherapeutic effects in human osteosarcoma. Carcinogenesis 33: 30-40, 2012

2. Fuchs B and Pritchard DJ: Etiology of osteosarcoma. Clin Orthop Relat Res 397: 40-52, 2002.

3. Gibbs CP Jr, Weber K and Scarborough MT: Malignant bone tumors. Instr Course Lect 51: 413-428, 2002.

4. Zhao Q, Wang C, Zhu J, Wang L, Dong S, Zhang G and Tian J: RNAi-mediated knockdown of cyclooxygenase 2 inhibits the growth, invasion and migration of Saos2 human osteosarcoma cells: a case control study. J Exp Clin Cancer Res 30: 26, 2011.

5. Mukherjee AK, Basu S, Sarkar N and Ghosh AC: Advances in cancer therapy with plant based natural products. Curr Med Chem 8: 1467-1486, 2001.
6. Smets LA: Programmed cell death (apoptosis) and response to anti-cancer drugs. Anticancer Drugs 5: 3-9, 1994.

7. Paschka AG, Butler R and Young CYF: Induction of apoptosis in prostate cancer cell lines by the green tea component, (-)-epigallocatechin-3-gallate. Cancer Lett 130: 1-7, 1998.

8. Kaufmann SH and Hengartner MO: Programmed cell death: alive and well in the new millennium. Trends Cell Biol 11: 526-534, 2001

9. Kaufmann SH and Earnshaw WC: Induction of apoptosis by cancer chemotherapy. Exp Cell Res 256: 42-49, 2000.

10. Reed JC: Apoptosis-regulating proteins as targets for drug discovery. Trends Mol Med 7: 314-319, 2001.

11. Banerjee M, Tripathi LM, Srivastava VM, Puri A and Shukla R: Modulation of inflammatory mediators by profen and curcumin treatment during chronic inflammation in rat. Immunopharmacol Immunotoxicol 25: 213-224, 2003.

12. Sahu RP, Batra1 S and Srivastava SK: Activation of ATM/Chk1 by curcumin causes cell cycle arrest and apoptosis in human pancreatic cancer cells. Br J Cancer 100: 1425-1433, 2009.

13. Wahl H, Tan L, Griffith K, Choi M and Liu JR: Curcumin enhances Apo2L/TRAIL-induced apoptosis in chemoresistant ovarian cancer cells. Gynecol Oncol 105: 104-112, 2007.

14. Goel A, Kunnumakkara AB and Aggarwal BB: Curcumin as 'Curecumin': from kitchen to clinic. Biochem Pharmacol 75: 787-809, 2008.

15. Anand P, Kunnumakkara AB, Newman RA and Aggarwal BB: Bioavailability of curcumin: problems and promises. Mol Pharm 4: 807-818, 2007

16. Adams BK, Cai J, Armstrong J, et al: EF24, a novel synthetic curcumin analog, induces apoptosis in cancer cells via a redoxdependent mechanism. Anticancer Drugs 16: 263-275, 2005.

17. Komatsu M, Kuroda M, Wang Y, et al: Manganese superoxide dismutase overexpression changes plating efficiency bidirectionally according to change in redox for SaOS2 human osteosarcoma cell line. Int J Oncol 26: 853-862, 2005.

18. Kim SG, Kim HH, Kim HK, et al: Differential expression and functional characterization of system $\mathrm{L}$ amino acid transporters in human normal osteoblast cells and osteogenic sarcoma cells. Anticancer Res 26: 1989-1996, 2006.

19. Hu W and Kavanagh JJ: Anticancer therapy targeting the apoptotic pathway. Lancet Oncol 4: 721-729, 2003.

20. Herrnring C, Reimer T, Jeschke U, et al: Expression of the apoptosis-inducing ligands FasL and TRAIL in malignant and benign human breast tumors. Histochem Cell Biol 113: 189-194, 2000 .

21. Li HJ, Wang CY, Mi Y, et al: FasL-induced apoptosis in bovine oocytes via the Bax signal. Theriogenology 80: 248-255, 2013.

22. Cheng YL, Lee SC, Lin SZ, et al: Anti-proliferative activity of Bupleurum scrozonerifolium in A549 human lung cancer cells in vitro and in vivo. Cancer Lett 222: 183-193, 2005.

23. Tian Z, Chen S, Zhang Y, et al: The cytotoxicity of naturally occurring styryl lactones. Phytomedicine 13: 181-186, 2006.

24. Hauser PJ, Han Z, Sindhwani P and Hurst RE: Sensitivity of bladder cancer cells to curcumin and its derivatives depends on the extracellular matrix. Anticancer Res 27: 737-740, 2007.

25. Kikuchi T, Pan X, Ishii K, et al: Cytotoxic and apoptosisinducing activities of 12-O-Acetylazedarachin $\mathrm{B}$ from the fruits of Melia azedarach in human cancer cell lines. Biol Pharm Bull 36: 135-139, 2013.

26. Martin SJ, Reutelingsperger CP, McGahon AJ, Rader JA, van Schie RCAA, LaFace DM and Green DR: Early redistribution of plasma membrane phosphatidylserine is a general feature of apoptosis regardless of the initiating stimulus: inhibition by overexpression of Bcl-2 and Abl. J Exp Med 182: 1545-1556, 1995.

27. Liu X, Zou H, Slaughter C and Wang X: DFF, a heterodimeric protein that functions downstream of caspase-3 to trigger DNA fragmentation during apoptosis. Cell 89: 175-184, 1997.

28. Ryu DS, Lee HS, Lee GS and Lee DS: Effects of the ethylacetate extract of Orostachys japonicus on induction of apoptosis through the p53-mediated signaling pathway in human gastric cancer cells. Biol Pharm Bull 35: 660-665, 2012.

29. Lavin MF and Gueven N: The complexity of p53 stabilization and activation. Cell Death Differ 13: 941-950, 2006.

30. Heath-Engel HM, Chang NC and Shore GC: The endoplasmic reticulum in apoptosis and autophagy: role of the BCL-2 protein family. Oncogene 27: 6419-6433, 2008.

31. Fisher DE: The p53 tumor suppressor: critical regulator of life \& death in cancer. Apoptosis 6: 7-15, 2001. 
32. Kluck RM, Bossy-Wetzel E, Green DR and Newmeyer DD: The release of cytochrome c from mitochondria: a primary site for Bcl-2 regulation of apoptosis. Science 275: 1132-1136, 1997.

33. Kluck RM, Esposti MD, Perkins G, et al: The pro-apoptotic proteins, Bid and Bax, cause a limited permeabilization of the mitochondrial outer membrane that is enhanced by cytosol. J Cell Biol 147: 809-822, 1999.

34. Yu CS, Huang AC, Lai KC, Huang YP, Lin MW, Yang JS and Chung JG: Diallyl trisulfide induces apoptosis in human primary colorectal cancer cells. Oncol Rep 28: 949-954, 2012.
35. Luo X, Budihardjo I, Zou H, Slaughter C and Wang X: Bid, a $\mathrm{Bcl} 2$ interacting protein, mediates cytochrome $\mathrm{c}$ release from mitochondria in response to activation of cell surface death receptors. Cell 94: 481-490, 1998.

36. Oltvai ZN, Milliman CL and Korsmeyer SJ: Bcl-2 heterodimerizes in vivo with a conserved homolog, Bax, that accelerates programmed cell death. Cell 74: 609-619, 1993. 